\title{
Some Notes on Granular Mixtures with Finite, Discrete Fractal Distribution
}

\author{
Emőke Imre ${ }^{1,2^{*}}$, István Talata ${ }^{3}$, Daniel Barreto ${ }^{4}$, Maria Datcheva $^{5}$, Wiebke Baille ${ }^{6}$, Ivan Georgiev ${ }^{7}$, \\ Stephen Fityus ${ }^{8}$, Vijay P. Singh ${ }^{9}$, Francesca Casini ${ }^{10}$, Giulia Guida ${ }^{10}$, Phong Q. Trang ${ }^{11,12}$, \\ János Lőrincz ${ }^{11}$ \\ 1 EKIK HBM Systems Research Center, Bánki Donát Faculty of Mechanical and Safety Engineering, Óbuda University, Bécsi út 96/B, \\ 1034 Budapest, Hungary \\ 2 AIAM Doctoral School, Óbuda University, Bécsi út 96/B, 1034, Budapest, Hungary \\ 3 Ybl Faculty, Óbuda University, Bécsi út 96/B, 1034, Budapest, Hungary \\ 4 School of Engineering and the Built Environment, Edinburgh Napier University, Merchiston Campus, EH10 5DT, Edinburgh, UK \\ 5 Institute of Mechanics, Bulgarian Academy of Sciences, Acad. G. Bonchev St., 1113 Sofia, Bulgaria \\ 6 Department of Civil and Environmental Engineering, Ruhr University Bochum, Universitätsstraße 150, 44801 Bochum, Germany \\ 7 Institute of Information and Communication Technologies, Bulgarian Academy of Sciences, Acad. G. Bonchev St., 1113 Sofia, Bulgaria \\ 8 School of Engineering, University of Newcastle, University Drive, NSW 2308, Callaghan, Australia \\ 9 Department of Biological and Agricultural Engineering, Texas A \& M University, Scoates Hall, Suite 321, 2117 TAMU, 333 Spence St, \\ College Station, TX 77843, USA \\ ${ }^{10}$ Department of Civil and Informatics Engineering, University of Roma Tor Vergata, Via del Politecnico, 1, 00133 Roma, Italy \\ ${ }^{11}$ Department of Engineering Geology and Geotechnics, Budapest University of Technology and Economics, Múegyetem rkp. 3, 1111 \\ Budapest, Hungary \\ 12 Bachy Soletanche, 126 Nguyen Thi Minh Khai St., Dist. 3, Ho Chi Minh City, Vietnam \\ * Corresponding author, e-mail: imre.emoke@uni-obuda.hu
}

Received: 20 August 2021, Accepted: 12 September 2021, Published online: 11 October 2021

\begin{abstract}
Why fractal distribution is so frequent? It is true that fractal dimension is always less than 3 ? Why fractal dimension of 2.5 to 2.9 seems to be steady-state or stable? Why the fractal distributions are the limit distributions of the degradation path? Is there an ultimate distribution? It is shown that the finite fractal grain size distributions occurring in the nature are identical to the optimal grading curves of the grading entropy theory and, the fractal dimension $\mathrm{n}$ varies between $-\infty$ and $\infty$. It is shown that the fractal dimensions 2.2-2.9 may be situated in the transitional stability zone, verifying the internal stability criterion of the grading entropy theory. Micro computed tomography $(\mu \mathrm{CT})$ images and DEM (distinct element method) studies are presented to show the link between stable microstructure and internal stability. On the other hand, it is shown that the optimal grading curves are mean position grading curves that can be used to represent all possible grading curves.
\end{abstract}

Keywords

grading curve, grading entropy, finite fractal distribution, degradation, breakage

\section{Introduction}

\subsection{Natural soils and fragmentation}

The fragmentation phenomena are described by a power law distribution [1]. The size distribution of fragments is

$$
N(\Delta \leq d) \sim d^{-n}
$$

where $N$ is the number of particles that have diameters $\Delta$ below the size $d$ (see the mass-based form in Appendix A). This fractal distribution law is independent of the material properties, the energy input and the relevant length scales. The value of negative exponent is called fractal dimension and is generally varying between 2.0 and 2.9 for 3-dimensional objects [1]. It will be shown in this paper that this values can be related to internally stable mixtures.

Fractal distributions are naturally occurring in granular materials, such as meteorites [2], gouge material [3, 4], avalanches, mines [5, 6] and in laboratory tests, like compression tests and ring-shear tests [7-9, 10-12]. The fractal dimension in some theoretical studies $[8,11]$ is around 2.5 . The grading data of monotonic tests reported in [12], presents values between 2.20 and 2.91 . 
In Space Mechanics, a fractal dimension $\sim 3$ is encountered for small particles at the planetary rings [1], while it is $\sim 6$ for large ones with size $\sim 0.1-1 \mathrm{~km}$.

\subsection{Aim and content of paper}

The question why fractal distributions and fractal dimensions 2.2-2.9 are so frequent in nature is analyzed in this paper revealing two controversal facts. To resolve the controversies, the concept of the internal stability rule of the grading entropy theory [13-19] is linked with the internal structure and degradation.

\section{Grading entropy}

\subsection{Space of grading curves}

In the grading curve measurement, the sieve hole diameters, the fraction limits are doubled. The measured grading curve is an empirical distribution function with finite, discrete distribution. An abstract cell system is defined with the diameter limits for the fractions (see Table 1, [18]):

$$
2^{j} d_{0} \geq d>2^{j-1} d_{0},
$$

where $d_{0}$ is the smallest diameter which may be equal to the height of the $\mathrm{SiO}_{4}$ tetrahedron $\left(\sim d_{0}=2^{-22} \mathrm{~mm}\right)$. The 2-base $\log$ of the diameter limits are integers, called abstract diameters. The relative frequencies of the fractions $x_{i}(i=1,2,3 \ldots, N)$ fulfil the following equation:

$$
\sum_{i=1}^{N} x_{i}=1, x_{i} \geq 0, \quad N \geq 1,
$$

where the integer variable $N$ is the number of the fractions between the finest and coarsest non-zero fractions.

The space of the grading curves with $N$ fractions is identified with the $N-1$ dimensional, closed simplex considering the relative frequencies $x_{i}$ the barycenter coordinates of the points. The vertices represent the fractions, the $2 \mathrm{D}$ edges are related to the two-mixtures, etc.

The sub-simplexes of a grading curve space are partly continuous, partly gap-graded. The continuous sub-simplexes have a lattice structure (Fig. 1(b)).

\subsection{Grading entropy parameters}

The grading entropy $S$ is derived from the statistical entropy concept, modified the original formula for unequal cells (since the fraction limits are doubled, [13, 17, 19]). Its result separates into two parts:

$S=S_{0}+\Delta S$,

where $S_{0}$ is base entropy and $\Delta S$ is entropy increment.
Table 1 Definition of fractions

\begin{tabular}{lcccc}
\hline$j$ & 1 & $\ldots$ & 23 & 24 \\
\hline Limits & $d_{0}$ to $2 d_{0}$ & $\ldots$ & $2^{22} d_{0}$ to $2^{23} d_{0}$ & $2^{23} d_{0}$ to $2^{24} d_{0}$ \\
$D_{j}$ or $S_{0 j}[-]$ & 1 & $\ldots$ & 23 & 24 \\
\hline
\end{tabular}

(a)

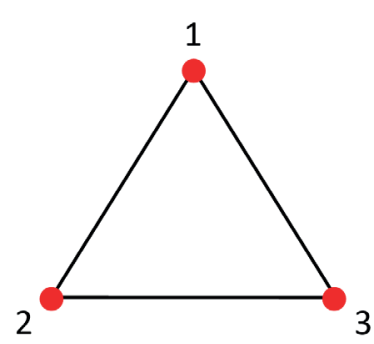

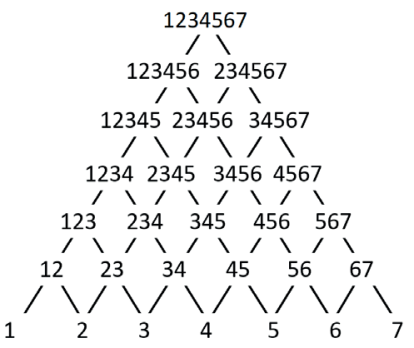

(b)
Fig. 1 Simplex images; (a) $N=3$, (b) $N=7$ represented by the lattice of continuous sub-simplexes (integers: fractions)

The base entropy is a kind of mean of the log diameter:

$S_{0}=\sum x_{i} S_{0 i}=\sum x_{i} i$,

since the $S_{0 k}$ - the $k$-th fraction entropy (Table 1) - expresses the power of 2 in the size of the statistical cell of $d$. The relative base entropy $A$ is defined as:

$A=\frac{S_{0}-S_{0 \min }}{S_{0 \max }-S_{0 \min }}$,

where $S_{0 \max }$ and $S_{0 \min }$ are the entropies of the largest and the smallest fractions, respectively. The relative base entropy varies between 0 and 1 . The entropy increment

$\Delta S=\frac{1}{\ln 2} \sum_{x_{i} \neq 0} x_{i} \ln x_{i}$,

expresses the statistical entropy due to the mixing of the various fractions. The normalized entropy increment $B$ is:

$B=\frac{\Delta S}{\ln N}$.

The normalized coordinates are not unique unless $N$ is defined properly (no zero fraction is allowed at the smallest or largest sides).

\section{Entropy diagrams}

\subsection{Entropy maps}

Four maps can be defined between the $N-1$ dimensional, open simplex (fixed $N$ ) and the two dimensional real Euclidean space of the entropy coordinates, the non-normalized $\Delta \rightarrow\left[S_{0}, \Delta S\right]$, normalized $\Delta \rightarrow[\mathrm{A}, \mathrm{B}]$, partly normalized $\Delta \rightarrow[\mathrm{A}, \Delta \mathrm{S}]$ or $\left[S_{0}, \mathrm{~B}\right]$. The map for fixed $N$ is continuous on the open simplex and can continuously be 
extended to the closed simplex. The entropy diagram image of the entropy map - is compact like the simplex (Fig. 2, [19-22]). It follows then, that the there is a maximum value for every value of A or $S_{0}$.

The inverse image of the regular values is a $N-3$ dimensional sphere, "centered" to the optimal point (Fig. 3). The inverse image of the conditional maximum $B$ for fixed $A$ (which is a critical value of the map, fixed $N$ and minimum diameter) is a single optimal point or unique optimal grading curve (Fig. 4).

\subsection{Optimal grading - mean grading curves}

The single optimal point or grading curve is defined as follows [18]:

$$
x_{1}=\frac{1}{\sum_{j=1}^{N} a^{j-1}}=\frac{1-a}{1-a^{N}}, x_{j}=x_{1} a^{j-1},
$$

where the parameter $a$ is the root of the equation:

$y=\sum_{j=1}^{N} a^{j-1}[j-1-A(N-1)]=0$.

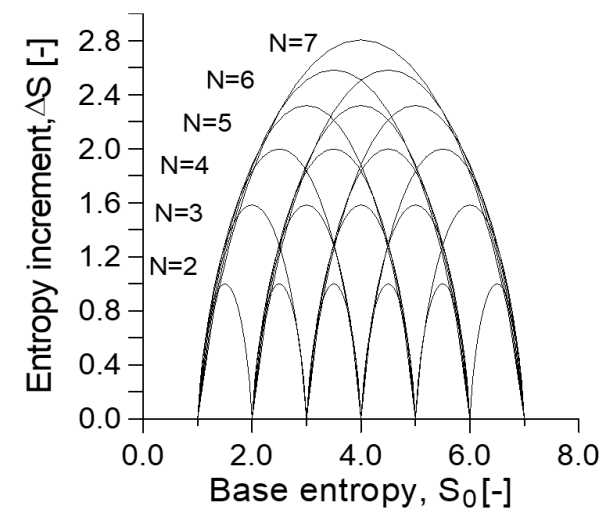

(a)

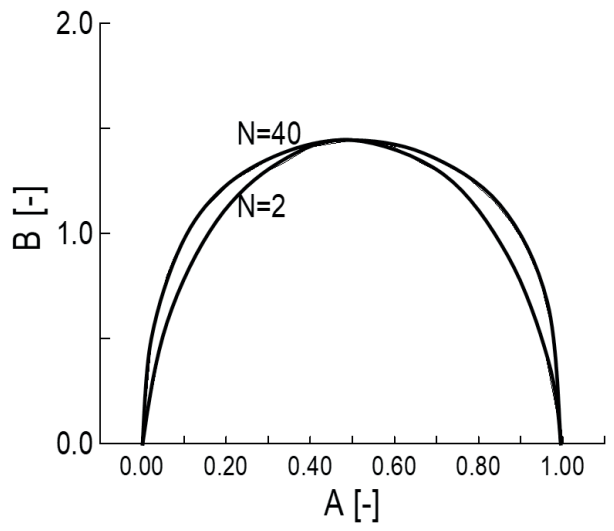

(b)

Fig. 2 Entropy diagram, the image of the optimal lines; (a): the nonnormalized entropy diagram, (b): the normalized entropy diagram

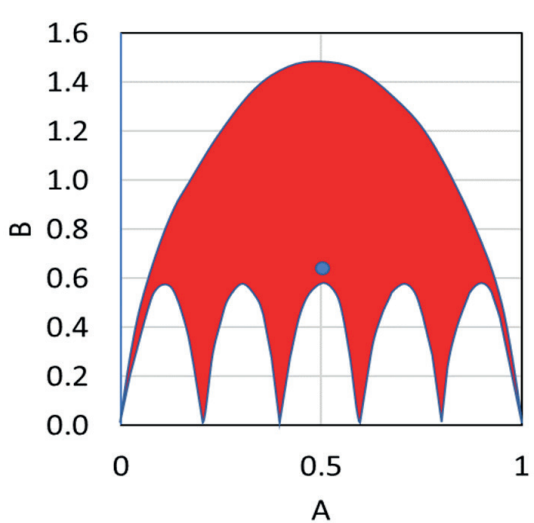

(a)

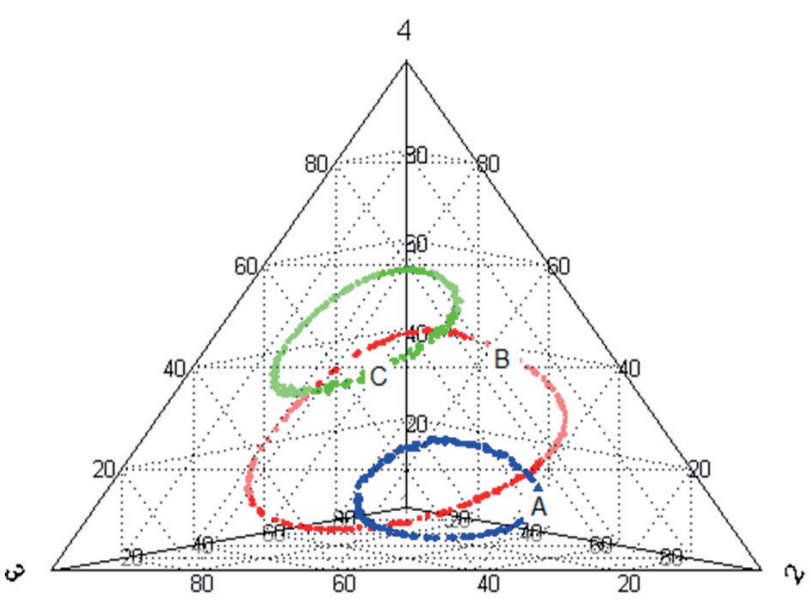

(b)

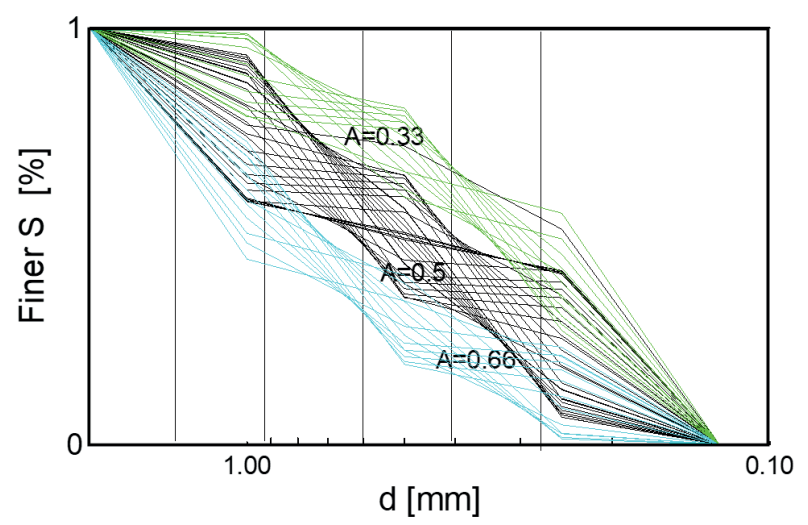

(c)

Fig. 3 The inverse image of three regular entropy diagram points (a) a regular value; (b) and (c) inverse image of $A=0.66, B=1.2$ (curve C), $A=0.5, B=1.2$ (curve B), $A=0.3, B=1.2$

The single positive root $a$ varies continuously between 0 and $\infty$ as $\mathrm{A}$ varies between 0 and $1, a=1$ at the symmetry point ( $A=0.5$ ) and $a>1$ on the $A>0.5$ side of the diagram (Appendices $\mathrm{C}, \mathrm{D}, \mathrm{E}$ ). The optimal grading curve is concave if $A<0.5$, linear if $A=0.5$ and convex if $A>0.5$ [19].

The maximum of the grading entropy $S$ is an optimal point where $a=2$, in the entropy diagram (Fig. 5) it is situated at varying $A$, depending on $N$. 


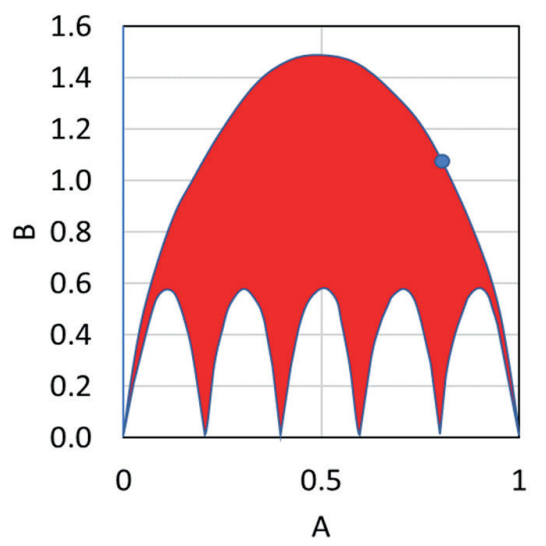

(a)

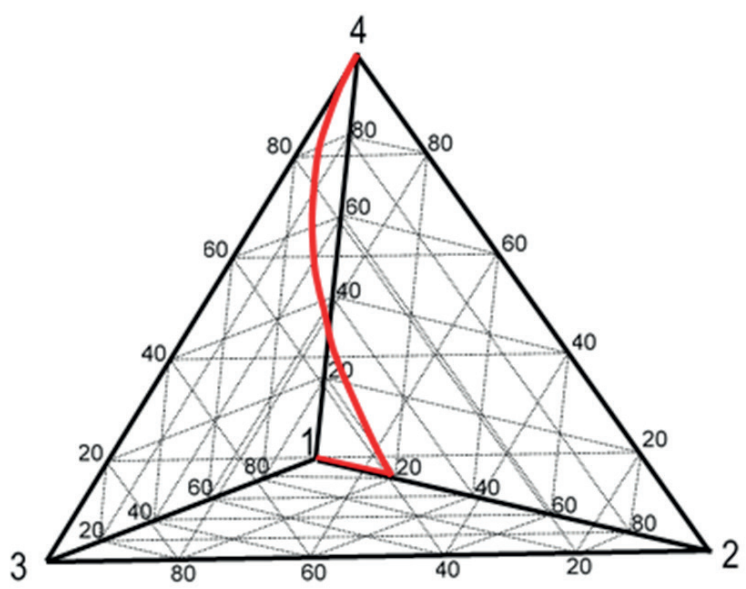

(b)

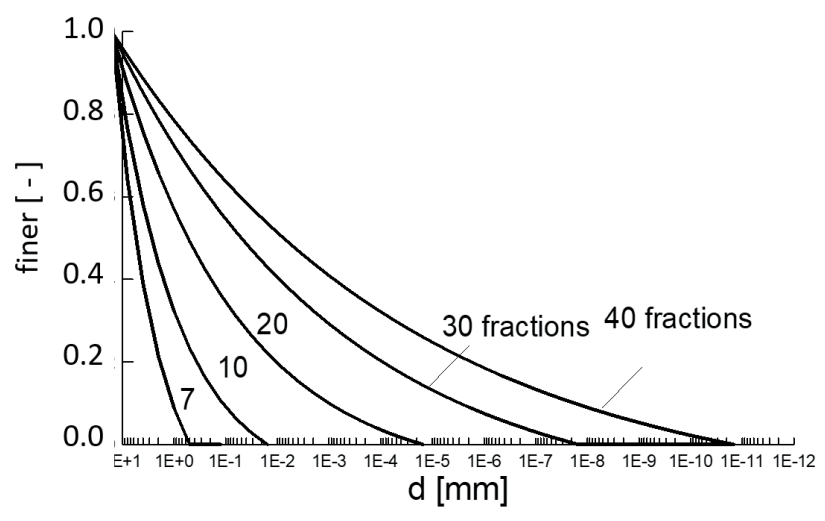

(c)

Fig. 4 The inverse image of the critical values; (a) a critical value, (b) the optimal line in simplex, $N=4$, (c) the optimal (fractal) grading curves, $A=2 / 3, N=7,10,20,30,40$

The subgraph area of all gradings is the same for a given A. The optimal grading curve has a geometric mean position (Fig. 6). The "distance" of a general grading depends on $B_{\max }-B$ for this given $A$ value (Appendix B).

\subsection{Link of optimal and fractal}

Instead of particle number, in a sieve analysis, the mass of all of the particles that are finer than the sieve mesh size $d$ is measured and we have [20] (Appendix A):

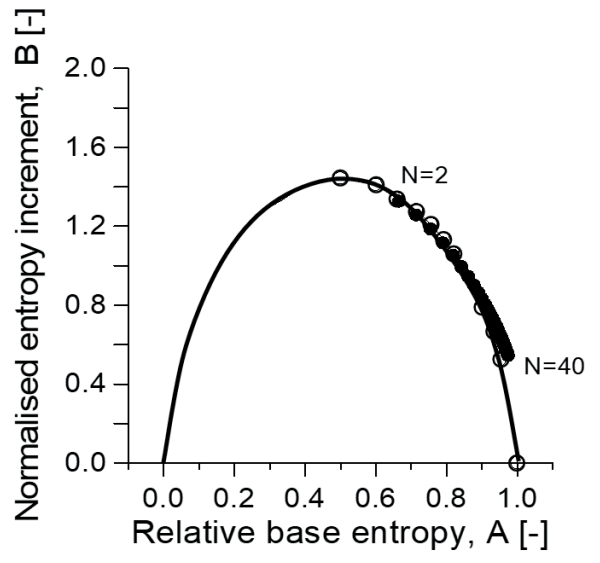

(a)

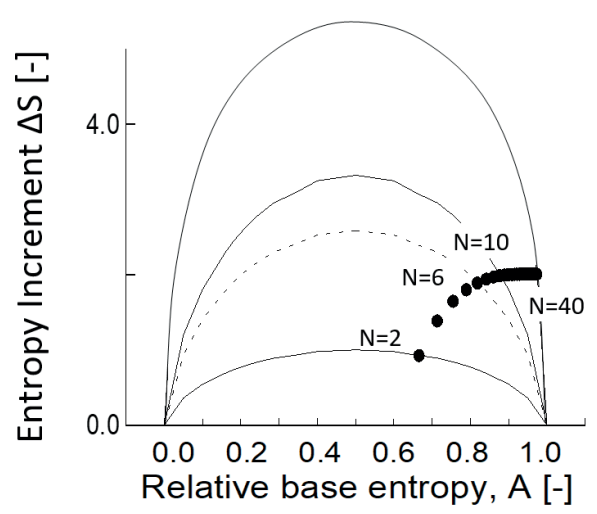

(b)

Fig. 5 The optimal grading curves related to $a=2$ (and $n=2$ ) giving the global maximum of $\mathrm{S}$ at $N=2$ to $N=40$, normalized and partly normalized diagrams

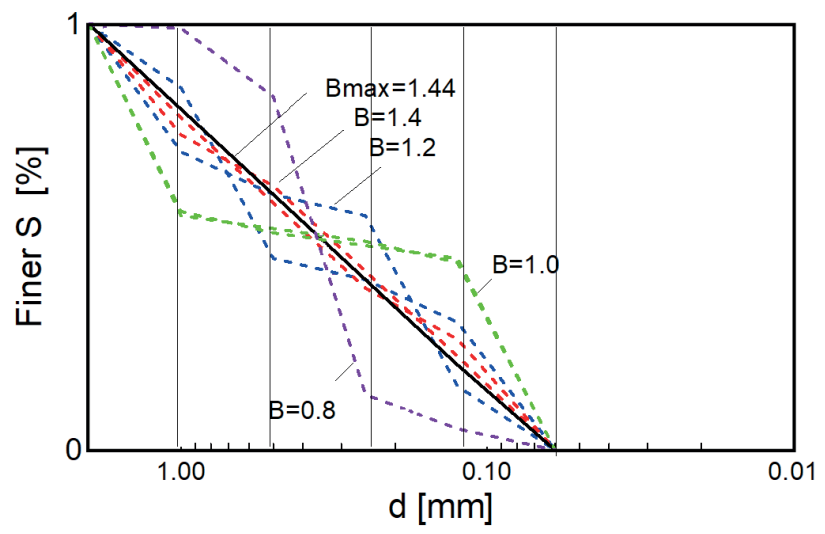

Fig. 6 Illustration of some gradings for $A=0.5$, the optimal line is the linear line. The subgraph area of all gradings is the same for a given $A$ if the grading curve is considered as a discrete distribution, the deviation from the optimal grading curve depends on $B_{\max }-B$. (Appendix B)

$M_{d}(\Delta \leq d)=\frac{d^{3-n}-d_{\min }^{3-n}}{d_{\max }^{3-n}-d_{\min }^{3-n}}$.

Taking into account that the fraction limits are defined by multiplying 2 , the relative frequencies of the fractions $x_{i}(i=1, \ldots, N)$ are as follows proving that the optimal grading curves are with finite fractal distribution: 
$x_{i}=\frac{d_{\min }^{3-n}}{d_{\max }^{3-n}-d_{\min }^{3-n}}\left(2^{3-n}-1\right)$,

$a=2^{3-n}$

and, therefore, the fractal dimension is:

$n=3-\frac{\log a}{\log 2}$

The fractal dimension $n$ varies between 3 and $-\infty$ on the $A>0.5$ side of the normalized diagram as a varies between 1 and $\infty$. The fractal dimension $n$ varies between 3 and $\infty$ on the $A<0.5$ side of the normalized diagram, as $a$ varies between 1 and 0 .

The $n$ value at a given $A$ value of depends on $N$ except at the symmetry point at $A=0.5$, where $B=1 / \mathrm{ln}^{2}, n=3, a=1$, Figs. 7-8.

4 Internal stability and comments on internal structure 4.1 Internal stability rule and structure

The criteria for grain structure stability (suffusion, piping) were elaborated on the basis of vertical water flow tests ("wash-out tests") where the soil was placed into a cylindrical permeameter $(20 \mathrm{~cm}$ height and $10 \mathrm{~cm}$ diameter

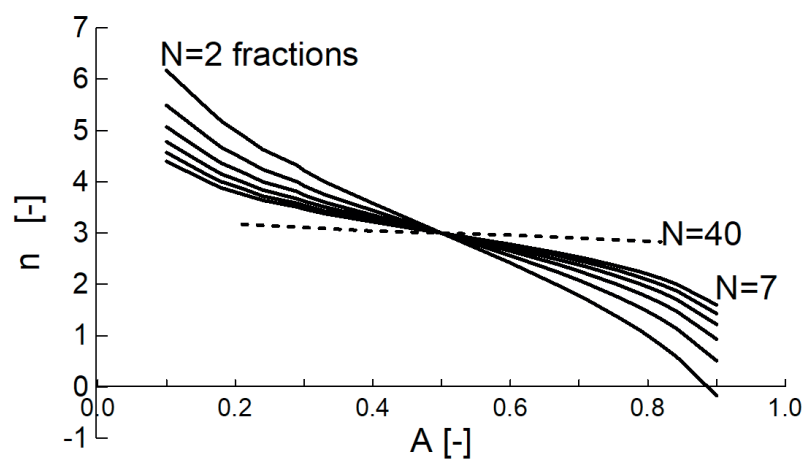

Fig. 7 The fractal dimension $n$ in terms of $A$ and $N$

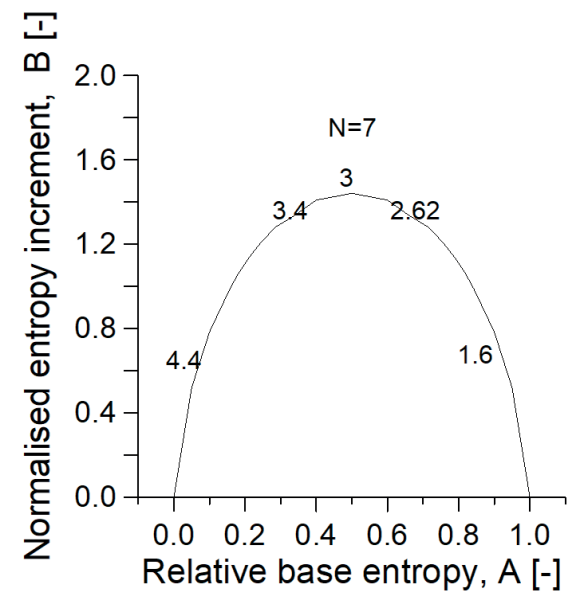

Fig. 8 Fractal dimension $n$ in terms of $A$ for $N=7$ at $A$ values of 0.1, $0.33,0.50,0.67,0.9$. For $N=2$ at the same $A$ values $n$ is $6,4,3,2,-0.17$ bounded at the bottom by a sieve. The bottom meshes permitted the passage of particles smaller than $1.2 \mathrm{~mm}$ but retain particles larger than $1.2 \mathrm{~mm}$. The applied downward hydraulic gradient, $i$, was as large as 4 or 5 .

In the zone "unstable" $(A<2 / 3)$ no internal structure of the large grains is present, the coarse particles "float" in the matrix of the fines and become destabilized when the fines are removed by erosion. In the zone "transition", the coarse particles start to form a stable skeleton if $N>2$. In the zone "stable", there is a structure of the larger particles which is inherently stable. Suffusion may occur in any zone on condition that the grading curves are (near) gapgraded with two missing fractions at least.

The division curve between zones "transition" and "stable" connects the maximum entropy points with fraction number less than $N$ (Fig. 9(a)). The $\mathrm{A}<2 / 3$ condition defines geometrically a hyperplane section of the simplex of the space of the grading curves.

The fractal distribution is stable if $n<2$ (independently of $N, A)$. The fractal distribution is internally unstable in terms of grading entropy criterion of if $A<2 / 3$. The fractal dimension $n$ at $A=2 / 3$ it is 2.62 for $N=7,2.25$ for $N=3$, 2.96 for $N=30$, see Table 2 .

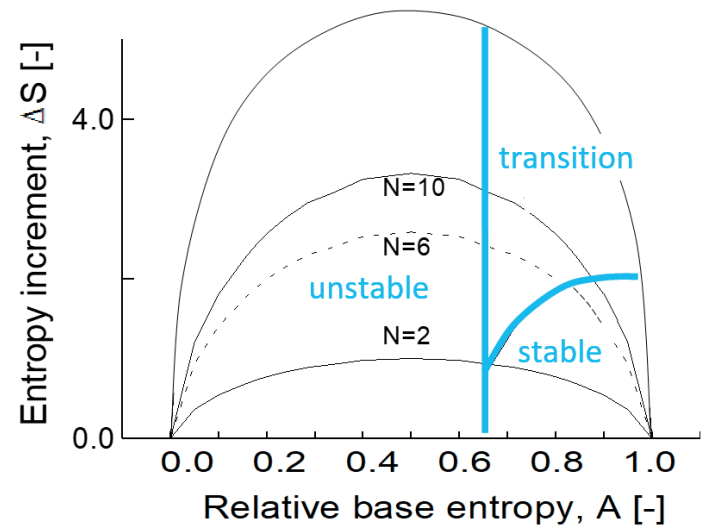

(a)

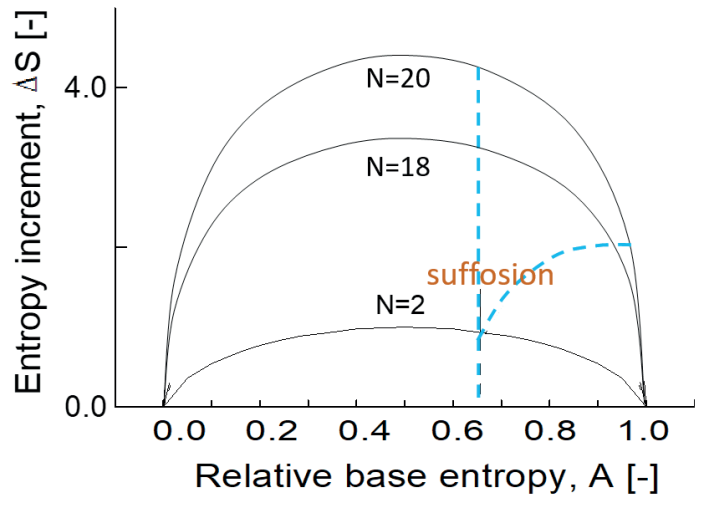

(b)

Fig. 9 Internal stability (a) and suffosion (b) criteria 
Table 2 The internal stability zones in terms of a fractal dimension (italic: unstable, bold: transition zone, normal: stable)

\begin{tabular}{lcccccc}
\hline$A[-] / N[-]$ & 2 & 3 & 4 & 5 & 6 & 7 \\
\hline 0.10 & 6.17 & 5.49 & 5.07 & 4.78 & 4.57 & 4.40 \\
0.66 & $\mathbf{2 . 0 0}$ & $\mathbf{2 . 2 5}$ & $\mathbf{2 . 4 0}$ & $\mathbf{2 . 4 9}$ & $\mathbf{2 . 5 7}$ & $\mathbf{2 . 6 2}$ \\
0.7 & 1.78 & $\mathbf{2 . 0 8}$ & $\mathbf{2 . 2 6}$ & $\mathbf{2 . 3 8}$ & $\mathbf{2 . 4 6}$ & $\mathbf{2 . 5 3}$ \\
0.71 & 1.68 & $\mathbf{2 . 0 0}$ & $\mathbf{2 . 1 9}$ & $\mathbf{2 . 3 3}$ & $\mathbf{2 . 4 2}$ & $\mathbf{2 . 4 9}$ \\
0.75 & 1.37 & 1.76 & $\mathbf{2 . 0 0}$ & $\mathbf{2 . 1 6}$ & $\mathbf{2 . 2 8}$ & $\mathbf{2 . 3 6}$ \\
0.79 & 1.09 & 1.54 & 1.81 & $\mathbf{2 . 0 0}$ & $\mathbf{2 . 1 3}$ & $\mathbf{2 . 2 4}$ \\
0.82 & 0.82 & 1.33 & 1.64 & 1.85 & $\mathbf{2 . 0 0}$ & $\mathbf{2 . 1 2}$ \\
0.84 & 0.58 & 1.13 & 1.47 & 1.70 & 1.87 & $\mathbf{2 . 0 0}$ \\
0.9 & -0.17 & 0.51 & 0.93 & 1.22 & 1.43 & 1.60 \\
\hline
\end{tabular}

As highlighted in $[12,18,23,24]$, the diagram is needed to be completed (Fig. 9(b)) for suffusion. It follows from the Terzaghi's filter rule that if the ratio between the minimum particle diameter of the filter and the maximum particle size of the base soil is smaller than 4, then particle migration may not occur. On the basis of this (and Kezdi's self-filtering theory) it is stated that in case of more than 2 missing fractions, suffosion may take place (Fig. 9).

\subsection{Internal microstructure}

The content of the internal stability criterion is as follows. The parameter $A$ expresses the proportion of the large grains as a pseudo-metric. If the large grains are present a large enough quantity, then they may form a stable skeleton. To verify this stability' rule some micro-level studies were performed.

\subsubsection{DEM study}

Preliminary 3D DEM simulations of spherical particles using periodic boundaries were performed within the opensource framework YADE. This code uses an explicit timecentered finite difference integration scheme to calculate the velocities and positions of all particles at every time step based on their accelerations resulting from Newton's second law. A Hertzian contact model in the normal contact direction was used (with Young's modulus, E $=70 \mathrm{GPa}$ and Poisson's ratio, $v=0.22$ ). In the shear direction a Coulombtype frictional law was used as routinely done in DEM research. Four fraction sizes were used to generate specimens of spheres: $0.125-0.25 \mathrm{~mm}, 0.25-0.5 \mathrm{~mm}, 0.5-1 \mathrm{~mm}$, and 1-2 $\mathrm{mm}$ (uniform distribution was assumed within the limits). 2-fraction soils $(N=2)$ were tested with various $A$ values under drained triaxial conditions until the critical state was achieved.
Table 3 Effect of A on the coordination number - DEM results

\begin{tabular}{ccc}
\hline$A$ & $Z$ & $Z_{\mathrm{m}}$ \\
\hline 0.10 & 2.87 & 4.32 \\
0.33 & 2.34 & 4.41 \\
0.50 & 2.52 & 4.42 \\
0.66 & 2.83 & 4.47 \\
0.90 & 3.39 & 4.47 \\
\hline
\end{tabular}

Each DEM specimen consisted of a different number of spheres. The rule of thumb used to determine specimen size was that every size fraction had to have at least 4000 particles to ensure statistically representative micro-scale data. All specimens were generated by generating a system of contactless spheres that was then isotropically consolidated to $200 \mathrm{kPa}$ with an inter-particle friction coefficient of 0.3 . As a result, specimens have a loose density that is close to minimum. The overall $(Z)$ and mechanical $\left(Z_{\mathrm{m}}\right)$ during critical state are shown in Table 3 . The coordination numbers represent the average number of contacts per particle for the specimen, the mechanical coordination number only includes those that are part of strong force chains (i.e., accounting for particles with more than two contacts).

Note that as $A$ increases, $Z_{\mathrm{m}}$ also increases indicating that the number of particles effectively transmitting stress increases. Furthermore, as $A$ increases, the difference between $Z$ and $Z_{\mathrm{m}}$ decreases. This implies that as $A$ increases the number of "rattlers" (i.e., particles that are not part of the strong force chains) reduces, the specimens become inherently more stable. Hence, there is a clear link between stability, relative base entropy and the mechanical coordination number.

\subsection{2 $\mu \mathrm{CT}$ study}

The internal structure is investigated using sand-fine powder mixtures with various fine content and results of various image techniques are reported in literature [21].

Analyzing $\mu \mathrm{CT}$ images of samples composed of sand fine powder mixtures showed that the number of contacts between the coarse particles decreased with the increase in the fines content (Fig. 10).

The $\mu \mathrm{CT}$ studies of 2 -fraction mixtures revealed that the microstructural meaning of the $A=2 / 3$ condition is the limit or the boundary of "coarse in fine" to "fine in course" configurations.

The stable structure is ensured such that the large grains form a skeleton. The gradual formation of the large grain skeleton for large fraction numbers needs some further research. 


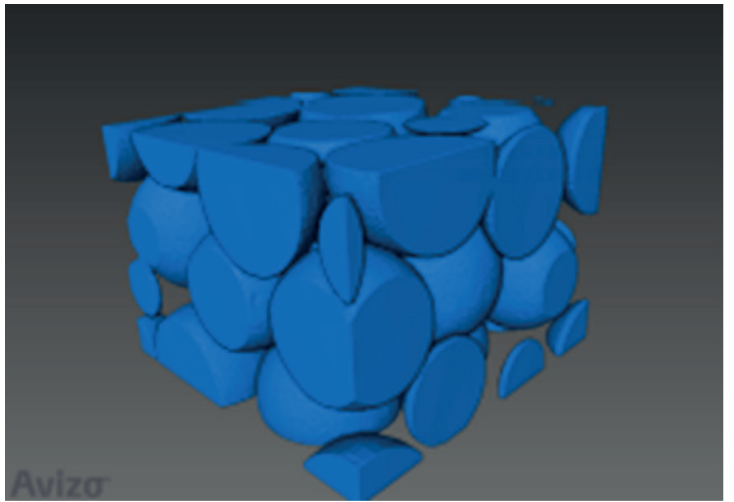

(a)

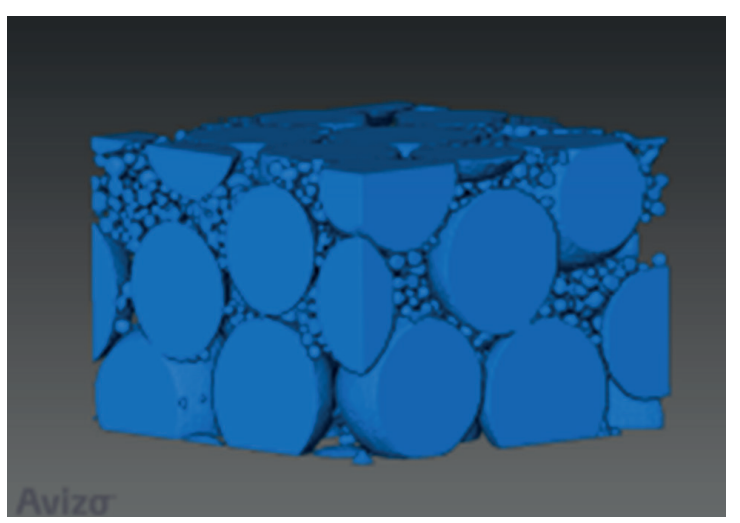

(b)

Fig. $10 \mu \mathrm{CT}$ scan image of clean granular matter with (a) no smalls, (b) $25 \%$ smalls [25]

\subsection{Probability of an internally stable grading curve}

The probability that an arbitrary $N$-fraction soil is not unstable can be characterized by the relative size of the grading curve space separated with the $A=2 / 3$ hyperplane on condition that the probability is the same in the whole simplex. The geometrical probability expressed by the ratio of the volume of the simplex of the grading curves where $A>2 / 3$ is met and the total volume of the whole simplex tends to be zero with $N$ (Table 4, [18]).

The probability is decreasing with the fraction number (e.g., for $N=2$, the $1 / 3$ part of the grading curve space is safe, for $N=3$, the $2 / 9$ part of the grading curve space is safe). Soils which meet both internal stability and segregation criterion $(7 / 10>A>2 / 3)$ may constitute very small part of the of the grading curve space and may need careful design in case of broadly graded filters.

Table 4 The geometrical probability that an arbitrary $N$-fraction grading curve is stable and segregation free

\begin{tabular}{lcccc}
\hline $\mathrm{N}[-]$ & 2 & 10 & 30 & 50 \\
\hline $\mathrm{P}(A>2 / 3)$ & $3.33 \mathrm{E}-01$ & $4.16 \mathrm{E}-02$ & $8.24 \mathrm{E}-04$ & $2.06 \mathrm{E}-05$ \\
$\mathrm{P}(0.7>A>2 / 3)$ & $3.33 \mathrm{E}-02$ & $2.36 \mathrm{E}-02$ & $7.54 \mathrm{E}-04$ & $2.03 \mathrm{E}-05$ \\
\hline
\end{tabular}

\section{Degradation path}

\subsection{Discontinuity of the entropy path if $N$ varies}

The grading curve of a granular matter may suddenly change due to breakage (appearing of new, finer fractions) or due to the addition of stabilization material like lime (disappearing of finer fractions and appearance of larger grains).

These sudden changes of the grading curve can be represented in the grading entropy diagram if $i(i=1,2 \ldots)$ zero fractions are added. Adding from the smaller diameter side, then the following discontinuity can be derived in the normalized entropy path (Fig. 11, [18]):

$$
\begin{aligned}
& B(N)-B(N+1)=\Delta S(N) \frac{1-\frac{\ln N}{\ln N+1}}{\ln N}, \\
& A(N+1)-A(N)=\frac{1-A(N)}{N} .
\end{aligned}
$$

The $A$ change for $i=1,2 \ldots$ additional larger zero fractions:

$A(N+1)-A(N)=\frac{1-A(N)}{N}$,

and, for $j=i / 2=1,2 \ldots$ zero fractions at both sides:

$$
A(N+i)-A(N)=\frac{1-2 A(N)) / 2}{N} .
$$

\subsection{Fragmentation path during breakage test}

The results of three kinds of crushing tests with two different rock materials developed in this research are presented in Fig. 12 and Fig. 13 [22, 25, 26].

The non-normalized entropy path is as follows. The entropy increment $\Delta S$ strictly monotonically increases (reflecting the increasing entropy principle of thermodynamics) and, the base entropy $S_{0}$ decreases (due to the decrease of the mean diameter Fig. 12).

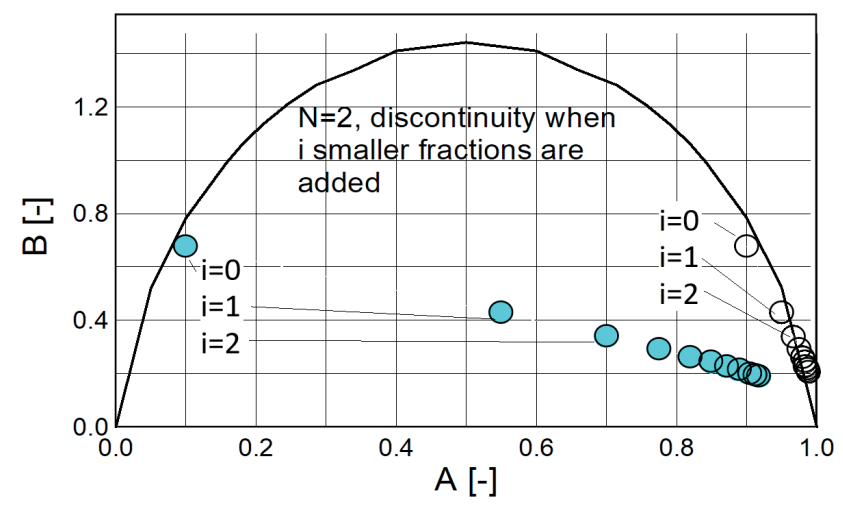

Fig. 11 Computed discontinuity, an initially $N=2$ optimal mixture, in the function of the increase in smaller fraction number (by adding zero fractions), initially convex and initially concave fractal gradings (blue) 


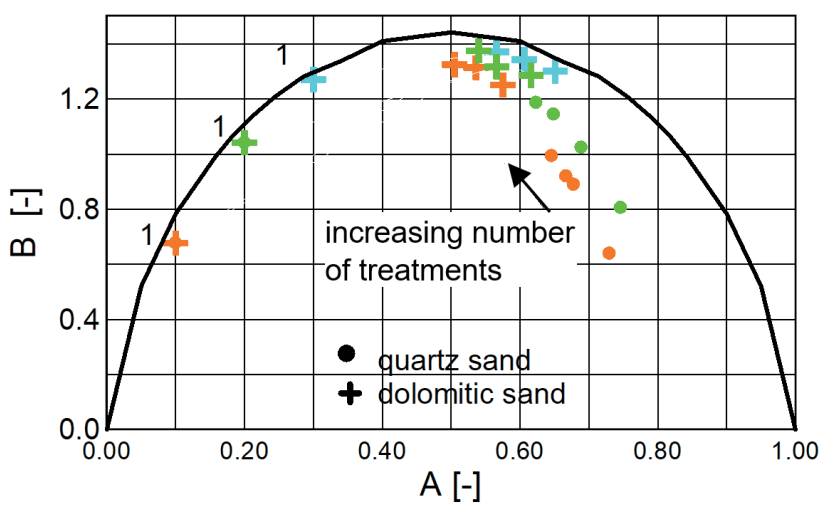

(a)

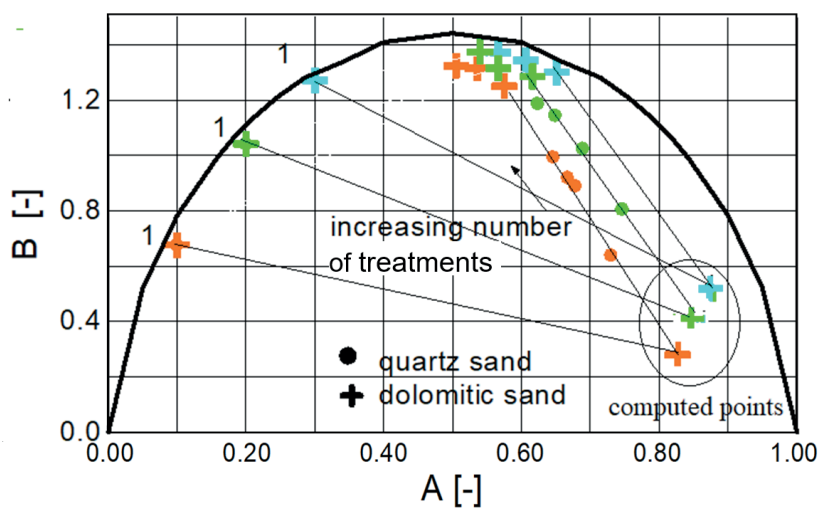

(b)

Fig. 12 Three crushing test results, two rocks, normalized diagram; (a) Measured data, (b) reevaluated data. The initial discontinuities (solid lines) are computed values. In the subsequent path (circles: silica sands, crosses: carbonate sands) every point indicates 10 crushing treatments

The normalized entropy path agrees with its non-normalized version for constant fraction number $N$ but is discontinuous if $N$ is changing.

When some new, smaller fractions appeared, the number of the fractions is increased at start of tests, then a 'discontinuity' appeared in the normalized diagram, which was computed with the suggested formula (Fig. 12). The jump size was directly related to $1-A$ toward the stable zone in parameter $A$, independently of the material.

In Fig. 12, the 1 indicates 3 initial 2-fraction gradings for both sands. When new fractions appear, the jump is also the same in the normalized entropy trajectory, the second, computed points are placed in the "stable" part.

The normalized entropy path was similar to the non-normalized one after the initial jump, being unique for a given load regime and initial grading (independently of the material properties of the solid). The velocity along the path was dependent on the material. The largest fraction did not disappear even for very large crushing treatments, as [20] indicates due to the "cushioning effect".

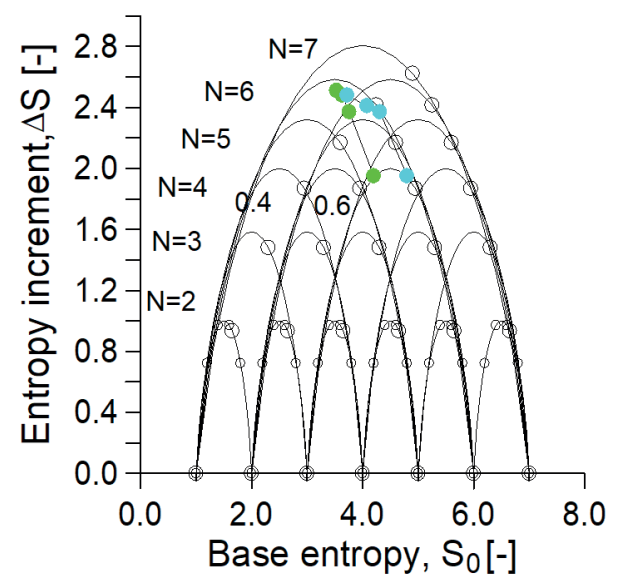

(a)

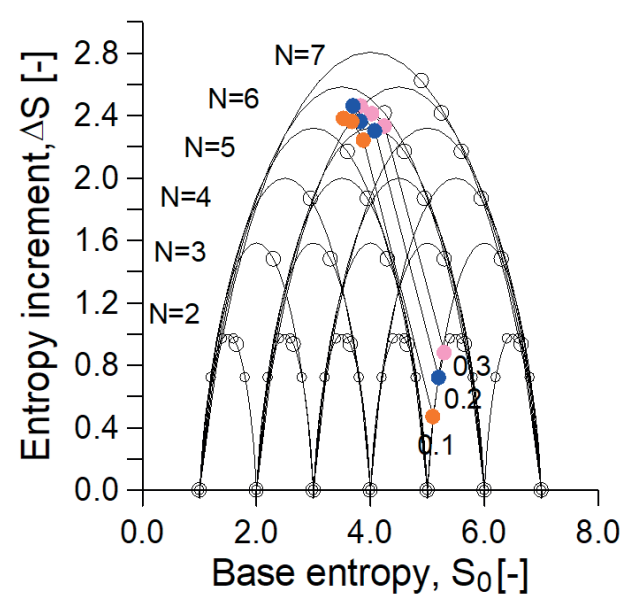

(b)

Fig. 13 Three crushing test results, two rocks, non-normalized diagram; (a) Silica sand - short paths, (b) carbonate sands - long paths sizes, every point indicates 10 additional crushing treatments along the line in upwards direction

\section{Degradation path - reevaluation of data}

Soil texture maturity was investigated in an open mine re-cultivation study (Fig. 14, Table 5, [27]). The project was undertaken over a short timeframe, within 10 weeks, using grading curve for texture characterization. The samples tested came from a coal mine where the mine owners had been rehabilitating the land progressively, so that there were areas that were rehabilitated 2 years ago, 8 years ago and 14 years ago. There were also soils which had formed on the same site naturally, over geological time (undisturbed).

The largest fraction at top may have been missing due to meteorological effects other than breakage (no 'cushioning effect' protected the large particles).

Most samples tested had an $A$ value equal to or larger than 0.666 , were not internally unstable. The samples tested were in the transitional stability zone, the only stable zoned 
sample was the undisturbed subsoil, the undisturbed top was less stable than this. The fractal dimension varied between 2.5 to 2.8 , with $N=17$.

The degradation was controlled by the increasing entropy increment (Fig. 14) and the decrease in diameter, in different rate for the two layers. According to the results, the key variables were the two top and sub layer differences, which increased with time, the top was more degraded. Interestingly, at the end of the construction work the entropy increment difference was about zero (due to mixing the soils everywhere, Table 5). However, the base entropy difference was larger than the undisturbed case.

The degradation path reflected the increasing entropy principle in terms of entropy increment (Fig. 14) and the decrease in diameter, similarly to the breakage test results.

The top-sub difference in $\Delta S$ was very small after construction (0.04) then it was monotonic increasing with time up to 0.16 since $\Delta S$ increased while $N$ was basically constant. Its tentative maximum can be the undisturbed value, 0.73 .

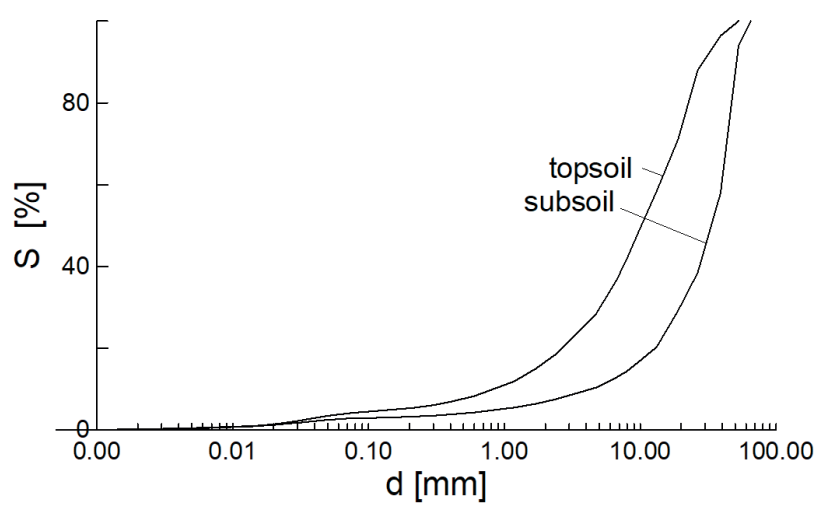

(a)

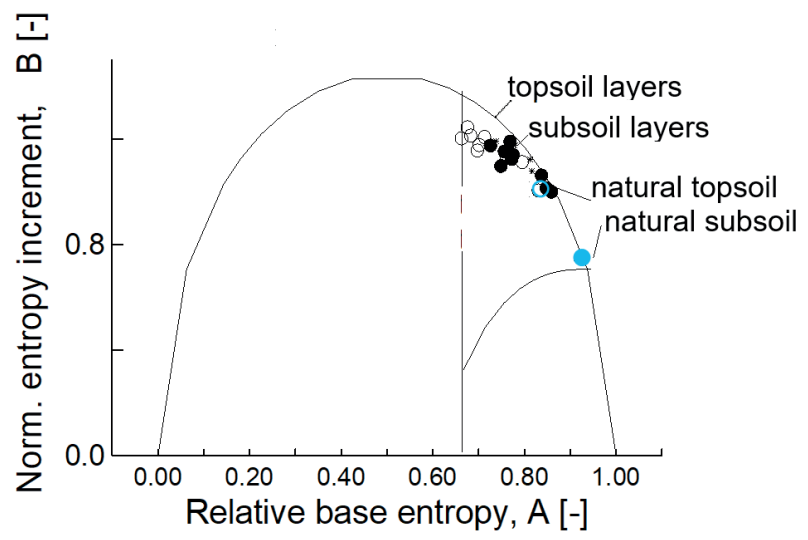

(b)

Fig. 14 The topsoil grading curves are more degraded. (a) Typical topsoil-subsoil gradings (b) Internal stability criterion in the $(N=17)$ normalized diagram, the degradation example of waste rock in open pit mine rehabilitation (topsoil samples in open symbols)
Table 5 The time evolution of the top and sub layers (reevaluation)

\begin{tabular}{lcc}
\hline$S_{\text {0base- }}-S_{0 \text { top }}[-]$ & $\Delta S_{\text {top- }}-\Delta S_{\text {base }}[-]$ & $t$ [year] \\
\hline 2.01 & 0.04 & 0 \\
2.11 & 0.18 & 8 \\
2.35 & 0.16 & 14 \\
$1.46^{*}$ & $0.73^{* *}$ & Undisturbed \\
\hline
\end{tabular}

$*$ small $S_{0 \text { base- }}-S_{0 \text { top }}$ value, $* *$ large $\Delta S_{\text {top- }}-\Delta S_{\text {base }}$ value

The sub-top difference $S_{\text {0base- }}-S_{0 \text { top }}$ increased from an initially larger value than the undisturbed state (originated possibly from segregation).

The topsoil-subsoil differences in terms of the entropy coordinates $S_{0}$ and $\Delta S$ were increasing with time similarly to soil maturity (Fig. 14, Table 5).

\section{Discussions}

\subsection{The material laws}

The empirical discrete grain size distribution curves with the same A have the same cubic sub-graph area (Appendix B) and the optimal curve has a kind of mean position, as shown in Fig. 6. Due to this, the fractal gradings are used to produce data in regressions between grading curves and soil parameters [17, 18].

Some test results made on fractal soils concerning the critical state friction angle and minimum dry density are shown in Fig. 15 [16, 19]. According to the results, the $A=2 / 3$ state is related to the maximum friction angle and the maximum value in the minimum dry density value. According to Table 3 , the $A=2 / 3$ state is related to maximum mechanical coordination number.

\subsection{The entropy path}

The effect of crushing and degradation is similar. The base entropy $S_{0}$ decreases, due to the decreasing of the grain size. The entropy increment $\Delta S$ is derived from the statistical entropy [28]. Since it is linked with a natural irreversible process, it reflects the entropy increasing principle [22].

Considering the normalized coordinates for fixed fraction number $N$, the relative base entropy $A$ decreases, the normalized entropy increment $B$ increases. As a result, the entropy path is moving towards the maximum normalized entropy increment line. When the entropy path reaches it then moves along the maximum normalized entropy increment line, where all points are related to fractal mixtures. Therefore, fractal distributions are formed by degradation in nature sooner or later. 


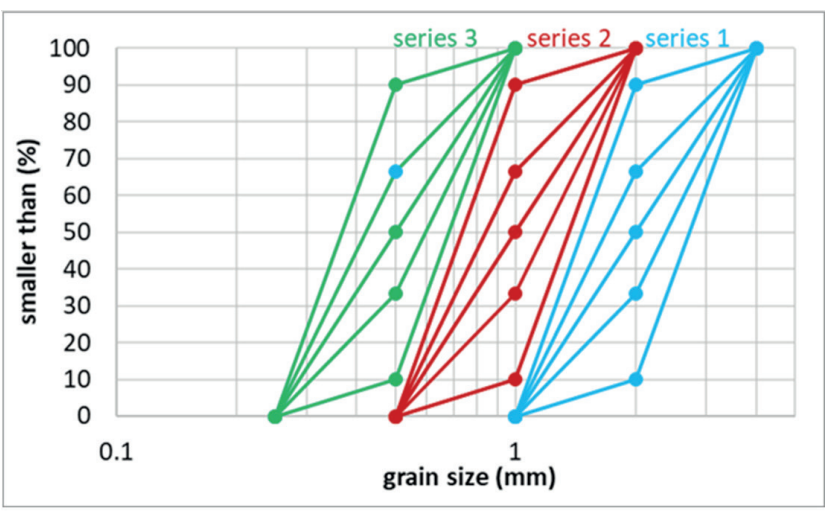

(a)

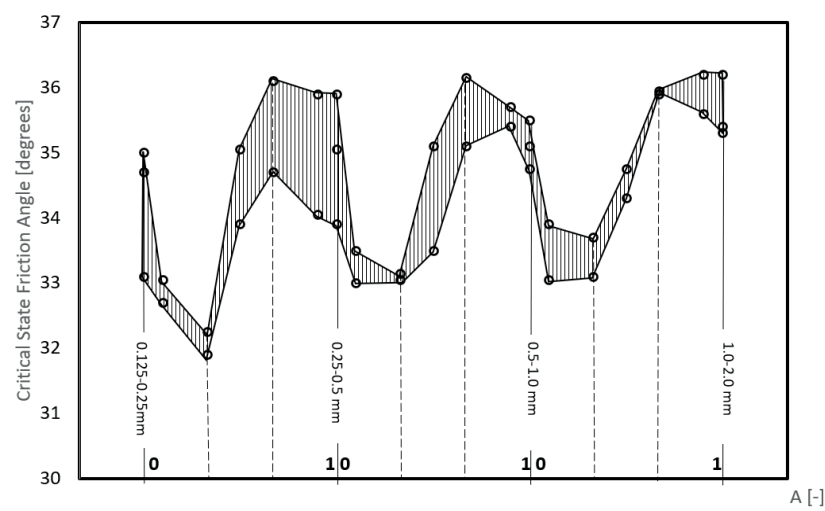

(c)

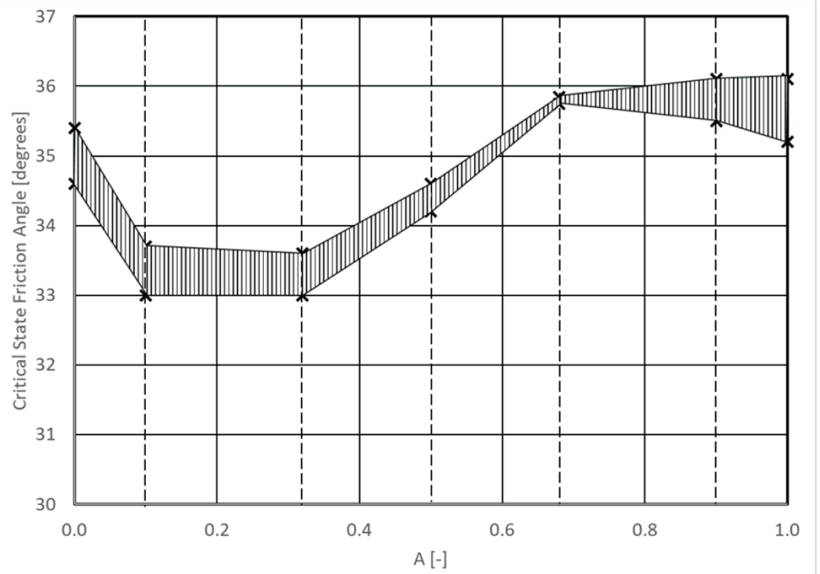

(b)

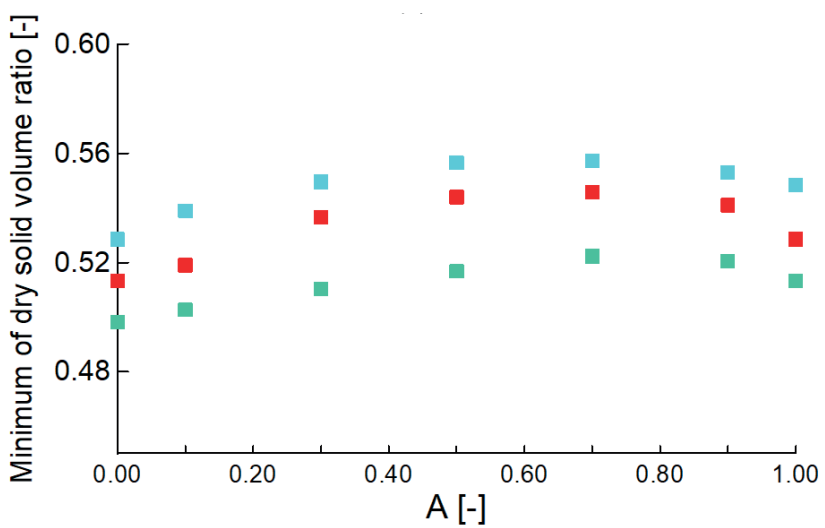

(d)

Fig. 15 Results of two kinds of tests on identical fractal grading curve series; (a) the tested grading curve series, (b) critical state friction angle from drained triaxial tests, 2-fractions mixtures with fractions of 1 to $2 \mathrm{~mm}$ and $2 \mathrm{~mm}$ to $4 \mathrm{~mm}$, (c) critical state friction angle from drained triaxial tests, a summary for all three series, 2 -fractions mixtures, (d) minimum solid volume ratio from minimum dry density test. The $A=2 / 3$ value seem to be the maximum value in both cases [23, 29-31]

However, the normalized entropy path is discontinuously drifted into the transitional stability zone or into the stable zone at the change of $N$ such that some new, smaller fractions appear. Therefore, stable distributions may be formed by degradation in nature.

\subsection{Ultimate distribution}

Is there any ultimate distribution? This question can tentatively be answered if some constraints during degradation are taken into account.

There is a 'cushion effect', the largest fraction may not disappear even at very large crushing treatments [20]. There is a crushing limit in terms of diameter, where the soil grains cannot further break [24].

Assuming these constraints, the final distribution is a kind of maximum entropy increment distribution. However, in case of weathering, the large grains may also disappear, taking into account this, the ultimate state can be a uniform distribution.

\section{Conclusions and summary}

\subsection{Questions}

\subsubsection{Fractal soils}

The finite fractal grain size distributions are the optimal grading curves. It follows that the fractal dimension $n$ may vary from minus to plus infinity, as the relative base entropy $A$ (normalized mean log scale diameter) varies between 0 and 1 , in the function of $N$.

At the symmetry point $A=0.5$, fractal dimension $n$ is 3 , independently of $N$. For large $N$ the fractal dimension $n$ is close to 3 over a wide range of $A$. On the $A>0.5$ side of the diagram $n<3$ and if $A<0.5$ then $n>3$ (Fig. 7, Fig. 8).

\subsubsection{The fractal dimension of natural soils}

The question why fractal distribution distributions with fractal dimensions 2.2-2.9 are so frequent in nature was analyzed in this paper. The main result is as follows.

The fractal dimension $n$ of the optimal grading curves varies between $-\infty$ to $+\infty$. However, the fractal dimension 
occurring in the nature is generally less than 3 . This controversy can be explained if the internal stability criterion of the grading entropy concept is taken into account.

The fractal distribution is stable if $n<2$ (independently of $N, A)$. The fractal distribution is internally unstable in terms of grading entropy criterion of if $A<2 / 3$. The fractal dimension $n$ at $A=2 / 3$ it is 2.62 for $N=7,2.25$ for $N=3$, 2.96 for $N=30$, see Table 2 . The transitional stability zone is situated between $A=2 / 3$ and an $A$ value depending on $N$, related to fractal dimension 2 .

The fractal dimensions of natural soils between around 2.2 to 2.96 are related to the transitional stability zone on condition that the $N$ is larger than about 6 . The fractal dimensions larger than 3 are internally unstable.

\subsubsection{Probability of a stable state for an arbitrary grading curve}

The internal stability criterion of the grading entropy concept can geometrically be defined as a $A<2 / 3$ part of the $N-1$ dimensional, closed simplex.

The probability of an arbitrary grain configuration to be the part of the stable domain was expressed by a geometrical probability. It was found that this was so small that that stable mixtures may hardy occur in nature by chance. The probability of a fractal soil is zero, also.

These controversies can be explained by the effect of fragmentation, which changes the grading entropy path either under the maximum entropy increment principle or - by the appearance of smaller fraction - into the direction of more stable states.

\subsection{The formation of fractal soils}

The question why internally stable fractal distribution distributions are so frequent in nature can be explained as follows.

According to the breakage test results, the entropy increment $\Delta S$ monotonically increases (reflecting the increasing entropy principle of thermodynamics) and, the base entropy $S_{0}$ decreases (due to the decrease of the mean diameter), resulting and upwards path.

Concerning the non-normalized diagram, the picture is not so simple. At constant $N$, the breakage path in the normalized path has similar upwards direction as the nonnormalized path, the internal stability is decreasing. When the path reaches the maximum normalized entropy increment line, then it proceeds along it, being realized through increasing fractal dimensions.
The value of the fractal dimension can theoretically be used to estimate the safety against internal un-stability in this part of the entropy path at constant $N$.

At the change of $N$ due to some new, smaller fractions, a jump can be found in the opposite direction, which can be computed by the suggested formulae which was validated by the breakage tests results.

Due to the new, smaller fractions, there is a sudden increase in the relative base entropy parameter $A$, the normalized entropy diagram point is drifted into a more stable part of the grading entropy diagram, the internal stability increases.

\subsection{Internal structure, material laws, further research}

A relationship between internal structure and the internal stability law was found in some DEM and $\mu \mathrm{CT}$ analyses. The internally more stable grain configurations seem to be characterized on a micro level by a larger mechanical coordinate number.

According to the results of some earlier measurements made on fractal gradings, the internal structure may influence the material laws as well.

Further research is suggested on many aspects. The connection of microstructure and internal stability is needed to be further investigated. The entropy path seems to be unique for a given loading breakage test and initial grading, its velocity depends on the material. The use of the normalized entropy path in material testing and stability analyses of natural slopes can be further investigated. The description of the parameters of constitutive equations using mean or fractal gradings can be explored. The entropy path is unknown if cushion effect is no more valid, the final state could theoretically be simulated by fractal iteration, where all fractions have a fractal distribution after additional changes.

\section{Acknowledgement}

We acknowledge the provided access to the infrastructure of the Laboratory for 3D Digitalization and Microstructure Analysis financially supported by the Science and Education for Smart Growth Operational Program (20142020) and the European Structural and Investment Fund through Grant No BG05M2OP001-1.001-0003. We also greatly acknowledge the fruitful discussions with Professor Itai Einav. 


\section{References}

[1] Kun, F. "Breakage of particles", Session introduction, presented at P\&G Conference, Montpellier, France, July, 3-7, 2017.

[2] Hartmann, W. K. "Terrestrial, lunar, and interplanetary rock fragmentation", Icarus, 10(2), pp. 201-213, 1969. https://doi.org/10.1016/0019-1035(69)90022-0

[3] Sammis, C. G., King, G., Biegel. R. "The kinematics of gouge deformations", Pure and Applied Geophysics, 125, pp. 777-812, 1987. https://doi.org/10.1007/BF00878033

[4] Airey, D .W., Kelly, R. B. "Interface behaviours from large diameter ring shear tests", In: Frost, J. D (ed.) Characterization and Behavior of Interfaces - Proceedings of Research Symposium on Characterization and Behavior of Interfaces, [e-book] IOS Press Ebooks, Amsterdam, The Netherlands, 2008, pp. 1-6. https://doi.org/10.3233/978-1-60750-491-7-1

[5] Imre, B., Laue, J., Springman, S. M. "Fractal fragmentation of rocks within sturzstroms: insight derived from physical experiments within the ETH geotechnical drum centrifuge", Granular Matter 12, pp. $267-285,2010$. https://oi.org/10.1007/s10035-009-0163-1

[6] McDowell, G. R., Bolton, M. D., Robertson, D. "The fractal crushing of granular materials", Journal of the Mechanics and Physics of Solids, 44(12), pp. 2079-2101, 1996.

https://doi.org/10.1016/S0022-5096(96)00058-0

[7] Palmer, A. C., Sanderson, T. J. O. "Fractal crushing of ice and brittle solids", Proceedings of the Royal Society A, 433(1889), pp. 469477, 1991.

https://doi.org/10.1098/rspa.1991.0060

[8] Steacy, S. J., Sammis, C. G. "An automaton for fractal patterns of fragmentation ", Nature, 353, pp. 250-252, 1991.

https://doi.org/10.1038/353250a0

[9] Roberts, J. E., de Souza, J. M. "The compressibility of sands", Proceedings - American Society for Testing Materials, 58, pp. 1269 1277, 1958.

[10] Hagerty, M. M., Hite, D. R., Ullrich, C. R., Hagerty, D. J. "OneDimensional High-Pressure Compression of Granular Media", Journal of Geotechnical Engineering, 119(1), pp. 1-18, 1991. https://doi.org/10.1061/(ASCE)0733-9410(1993)119:1(1)

[11] Turcotte, D. L. "Fractals and fragmentation", JGR Solid Earth, 91(B2), pp. 1921-1926, 1986. https://doi.org/10.1029/JB091iB02p01921

[12] Miao, G., Airey, D. "Breakage and ultimate states for a carbonate sand", Géotechnique, 63(14), pp 1221-1229, 2013. https://doi.org/10.1680/geot.12.P.111

[13] Lőrincz, J. "Talajok szemeloszlási entrópiája" (Soils grading entropy), PhD Thesis, Budapest University of Technology and Economics, 1986. (in Hungarian)

[14] Imre, E., Lőrincz, J., Trang, Q. P., Fityus, S., Pusztai, J., Telekes, G., Schanz, T. "A general dry density law for sands", KSCE Journal of Civil Engineering, 13(4), pp. 257-272, 2009. https://doi.org/10.1007/s12205-009-0257-7

[15] Imre, E., Talata, I. "Some comments on fractal distribution", In: Proceedings of MAFIOK 2017, Budapest, Hungary, 2017, pp. $22-32$.
[16] Imre, E., Nagy, L., Lőrincz, J., Rahemi, N., Schanz, T., Singh, V. P., Fityus, S. "Some Comments on the Entropy-Based Criteria for Piping", Entropy, 17(4), pp. 2281-2303, 2015.

https://doi.org/10.3390/e17042281

[17] Lörincz, J., Imre, E., Fityus, S., Trang, P. Q., Tarnai, T., Talata, I. Singh, V. P. "The Grading Entropy-Based Criteria for Structural Stability of Granular Materials and Filters", Entropy, 17(5), pp. 2781-2811, 2015. https://doi.org/10.3390/e17052781

[18] Imre, E., Baretto, D., Talata, I., Baille, W., Rahemi, N., Goudarzy, M., Lörincz, J., Singh, V. P. "Grading curves and internal stability", In: Proceedings of MAFIOK 2019, Dunaújváros, Hungary, 2019, pp. 116-128.

[19] Lörincz, J., Imre, E. M., Singh, V. P. "The Grading Entropy-Based Criteria for Structural Stability of Granular Materials and Filters", In: Sakellariou, M. (ed.) Granular Materials, InTech Open, Rijeka, Croatia, 2017, pp. 162-182. https://doi.org/10.5772/intechopen.69167

[20] Einav, I. "Breakage mechanics - Part I: Theory", Journal of the Mechanics and Physics of Solids, 55(6), pp. 1274-1297, 2007. https://doi.org/10.1016/j.jmps.2006.11.003

[21] Goudarzy, M. "Micro and Macro Mechanical Assessment of Small - Intermediate Strain Properties of Granular Material", PhD Thesis, Ruhr University Bochum, 2015.

[22] Lörincz, J., Imre, E., Gálos, M., Trang, Q. P., Rajkai, K., Fityus, I., Telekes, G. "Grading Entropy Variation Due to Soil Crushing", International Journal of Geomechanics, 5(4), pp. 311-320, 2005. https://doi.org/10.1061/(ASCE)1532-3641(2005)5:4(311)

[23] Imre, E., Lorincz, J., Trang, P. Q., Csonka, I., Kaczvinszki-Szabó, V., Telekes, G., Goudarzy, M., Rahemi, N., Baille, W., Schanz, T., ..., Singh, V. P. "Preliminary study on the relationship between dry density of sands and the grading entropy parameters", In: Proceedings of the XVII European Conference on Soil Mechanics and Geotechnical Engineering, XVII ECSMGE 2019, Reykjavík, Iceland, 2019, pp. 996-1002.

[24] Kendall, K. "The impossibility of comminuting small particles by compression", Nature, 272, pp. 710-711, 1978. https://doi.org/10.1038/272710a0

[25] Imre, E., Trang, P. Q., Fityus, S., Casini, F., Guida, G., Lőrincz, J. "Grading entropy and breakage of Granular Matter", presented at 14th Joint European Thermodynamics Conference, JETC 2017, Budapest, Hungary, May, 21-25, 2017.

[26] Fityus, S., Imre, E. "The significance of relative density for particle damage in loaded and sheared gravels", In: Powders and Grains 2017 - 8th International Conference on Micromechanics on Granular Media, EPJ Web Conference, 140, Article number: 07011, 2017. https://doi.org/10.1051/epjconf/201714007011

[27] Imre, E., Fityus, S. "The use of soil grading entropy as a measure of soil texture maturity", In: Proceedings of the XVI Danube - European Conference on Geotechnical Engineering, Skopje, Republic of North Macedonia, 2018, pp. 639-644.

[28] Korn, G. A., Korn, T. M. "Mathematical Handbook for Scientists and Engineers", 2nd eds., McGraw-Hill Book Company, New York, NY, USA, 1975. 
[29] Imre, E., Hazay, M., Juhász, M., Lőrincz, J., Rajkai, K., Schanz, T., Lins, Y., Hortobagyi, Zs. "Sand mixture density", Proceedings of Sixth International Conference on Unsaturated Soils, UNSAT 2014, Sydney, Australia, 2014, pp. 691-697.

[30] Göblyös, "The dependence of shear friction angle on the entropy parameters", MSc Thesis, Budapest University of Technology and Economics, 1989

\section{Appendix A}

\section{Fractal distribution on mass base}

The derivation of a mass-based distribution from a particle number based is as follows ([26]). In a fractal granular medium, the number of the collection of particles that have diameters $\Delta$ below the size $d$ is expressed by (see e.g., in [26])

$$
N(\Delta \leq d)=C d^{-\alpha},
$$

where $C$ is a constant of proportionality and, $\alpha=-n, n$ is the fractal dimension. In a sieve analysis, the mass of all of the particles that are finer than the sieve mesh size $d$ is normally measured, i.e.,

$$
M_{d}(\Delta \leq d)=\int_{d_{m}}^{d} s \rho \Delta^{3} \mathrm{~d} N(\Delta),
$$

where $s$ denotes the shape factor, which for spheres becomes $\pi / 6$, and $\rho$ is the specific mass. Differentiating:

$\mathrm{d} N(\Delta)=C \alpha\left(\Delta^{\alpha-1}\right) \mathrm{d} \Delta$

so that

$$
M_{d}(\Delta \leq d)=\frac{C s \alpha \rho}{3-\alpha}\left(d^{3-n}-d_{\min }^{3-n}\right) .
$$

The grain size cumulative distribution by mass is given by dividing with the total mass:

$$
\frac{M_{d}(\Delta \leq d)}{M_{t}}=\frac{d^{3-n}-d_{\min }^{3-n}}{d_{\max }^{3-n}-d_{\min }^{3-n}}
$$

\section{Appendix B}

\section{Subgraph area at fixed $A$}

The relative base entropy $A$ :

$$
A=\frac{\sum_{i=1}^{N} x_{i}\left(S_{0 i}-S_{0 \min }\right)}{N-1}=\frac{\sum_{i=1}^{N} x_{i}(i-1)}{N-1},
$$

is related to subgraph area of discrete distribution curve in terms of $D$. The area of each rectangle is equal to the ordinate assuming identical base. The total area:
[31] Barreto, D., Imre, E. "Preliminary study on the relationship between statistical entropy coordinates and the mechanical behaviour of granular materials", presented at 14th Joint European Thermodynamics Conference, JETC 2017, Budapest, Hungary, May, 21-25, 2017

$$
\begin{aligned}
& T_{s u b}=\sum_{\mathrm{i}=1}^{1} x_{i}+\sum_{\mathrm{i}=1}^{2} x_{i} \ldots+\sum_{\mathrm{i}=1}^{\mathrm{N}-1} x_{i}=\sum_{\mathrm{j}=2}^{\mathrm{N}}\left(1-\sum_{\mathrm{i}=\mathrm{j}}^{\mathrm{N}} x_{i}\right)= \\
& =N-1-\sum_{\mathrm{i}=1}^{\mathrm{N}} x_{i}(i-1)=(N-1)[1-A] .
\end{aligned}
$$

The mean ordinate $y_{\text {mean }}=T_{\text {sub }} /(N-1)$ is measuring the area below the distribution curve (Fig. 16). Since $A=1-y_{\text {mean }}, A$ is the complement to the subgraph area. The $A$ expresses the distance from the smallest fraction.

\section{Appendix C}

\section{Uniqueness of the optimal grading curves}

In this section a polynomial $y$ (see Section 3.2) and its single positive root $a$ are analyzed. By dividing the polynomial $y$ with $\left(-a^{N-1}\right)$, the symmetry appears:

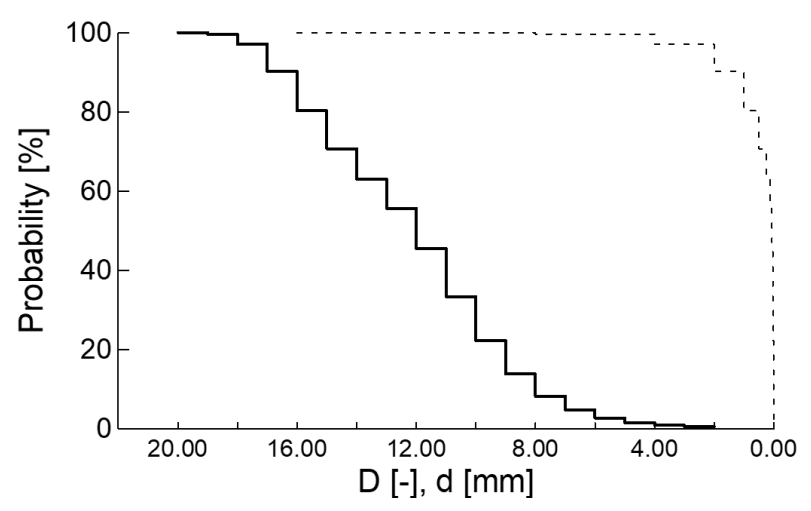

(a)

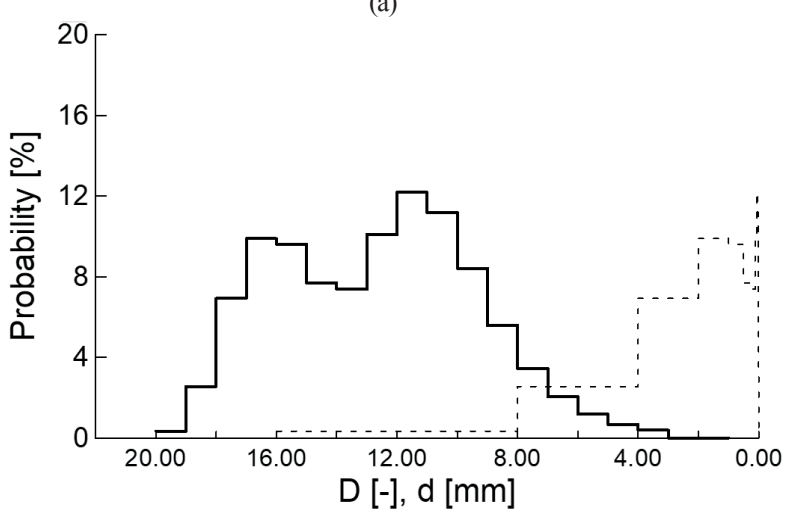

(b)

Fig. 16 Discrete representations of (a) measured cumulative and (b) density PSD. The area of each rectangle is equal to the ordinate 
$y_{0}=\sum_{j=1}^{N} \frac{1}{a^{N-j}}[N-j-(1-A)(N-1)]=0$.

Using the substitution of $a=e^{x}$ the following can be derived: (26)

$\left[\cosh \frac{(N+1) x}{2}-\cosh \frac{(N-1) x}{2}\right](1-2 A)$
$+\left[\sinh \frac{(N+1) x}{2}-\sinh \frac{(N-1) x}{2}\right]-\frac{2}{N-1} \sinh \frac{(N-1) x}{2}=0$,

$y_{2}=\frac{1}{N-1}\left[N \operatorname{coth} \frac{N x}{2}-\operatorname{coth} \frac{x}{2}\right]+(1-2 A)=y_{3}+(1-2 A)=0$.

Function $y_{3}$ is odd and the further analysis is presented only for positive values of the independent variable. Its limit is at plus infinity is 1 , it is continuously differentiable with a positive derivative. Having a continuous and positive derivative, it follows from the application of the local homeomorphism theorem that $y_{3}$ has a continuous inverse. No closed form solution for the inverse of $y_{3}$ is available.

It can be concluded that as $A$ varies within its range $[0,1], a$ varies continuously between 0 and $\infty$ having some symmetry.

\section{Appendix D}

\section{Closed form formula for maximum $B$ line}

The maximum $B$ lines for various $N$ are shown in Fig. 2, in the symmetry axis of the diagram they coincide, and nearly coincide otherwise as follows:
$A=\frac{1-a}{1-a^{N}} \frac{\sum_{i=1}^{N} a^{i-1}(i-1)}{N-1}, a \neq 1$,

$$
B=-\frac{1}{\ln N \ln 2}\left[\ln \frac{1-a}{a-a^{N}}+\ln a^{-\frac{a}{a-1} \frac{(N-1) a^{N}-N a^{N-1}+1}{(N-1) a^{N}-N+1}(N-1)}\right] .
$$

\section{Appendix E}

\section{Closed form formula for $\mathrm{A}$ in terms of $\mathrm{a}$ and $\mathrm{N}$}

Let

$f(x)=\sum_{i=1}^{N} x^{i-1}=\frac{x^{N}-1}{x-1}$,

then

$$
\begin{aligned}
& x f^{\prime}(x)=x \sum_{i=1}^{N}(i-1) x^{i-2}=x\left(\frac{x^{N}-1}{x-1}\right)^{\prime} \\
& =\frac{(N-1) x^{N+1}-N x^{N}+x}{(x-1)^{2}} .
\end{aligned}
$$

$$
\text { Since } \sum_{i=1}^{N}(i-1) a^{i-1}=A(N-1) \sum_{i=1}^{N} a^{i-1} \text {, }
$$

so af $(a)=A(N-1) f(a)$ and thus:

$$
A=\frac{a}{N-1} \frac{f^{\prime}(a)}{f(a)}=\frac{a}{a-1} \frac{(N-1) a^{N}-N a^{N-1}+1}{(N-1) a^{N}-N+1} .
$$

